\title{
REVISIONES
}

\section{La lectura mediada de literatura infantil como herramienta para la alfabetización emocional}

\author{
The mediated reading of child literature as a tool \\ for the basic emotional literacy \\ A leitura mediada da literatura infantil como \\ ferramenta para a alfabetização emocional
}

\author{
Enrique Riquelme*, Felipe Munita** \\ * Carrera de Pedagogía Básica Intercultural en Contexto Mapuche. Escuela de Educación Básica. \\ Facultad de Educación. Universidad Católica de Temuco. E-mail: eriquelme@uct.cl \\ ** Carrera de Pedagogía Básica con Especialización. Escuela de Educación Básica. \\ Facultad de Educación. Universidad Católica de Temuco. E-mail: fmunita@uct.cl \\ Dirección postal: Casilla 15 D, Facultad de Educación, U. Católica de Temuco.
}

\begin{abstract}
RESUMEN
La lectura de literatura en la infancia permite al niño lector explorar mundos de ficción en los que se representan distintas realidades caracterizadas por una serie de interacciones sociales y, por ende, por procesos emocionales. En el presente artículo, los autores destacan los aportes de la lectura de literatura infantil en el desarrollo emocional y social de los niños, a través del proceso denominado "lectura mediada". En este proceso, el adulto lector opera como un mediador de la experiencia emocional "ficcional" de los personajes en la narración, del relato y aquellos procesos reales que caracterizan el contexto y la experiencia vital de los niños, sin que la base de la narración sea direccionarlos para "enseñar" cierta emoción. De esta forma, la lectura mediada de literatura en la infancia opera como una herramienta de alfabetización emocional, proceso que permite el reconocimiento de emociones en sí mismo y en los otros, siendo esta la base de la empatía y conducta prosocial. El artículo finaliza discutiendo los alcances del proceso de lectura mediada de literatura infantil como una herramienta que favorece la inclusión escolar, y permite la interacción entre los aspectos cognitivos y afectivos en la educación formal.
\end{abstract}

Palabras clave: literatura infantil, lectura mediada, alfabetización emocional.

\begin{abstract}
The reading of literature during childhood allows the child reader to explore the world of fiction in which different realities are characterized by a series of social interactions and, therefore, by emotional processes. In the present article, the authors highlight the way in which the reading of child literature contributes to the emotional and social development of the children through a process named "mediated reading". During this process, the adult reader operates as a mediator of the emotional "fictional" experience of the characters of the narration, of the tale and of those real processes that characterize the context and the vital experience of the children. Furthermore, the narration is not intended to draw the children into the "teaching" of a specific emotion. In this way, the mediated reading of literature during childhood operates as a tool of emotional literacy, a process that allows the recognition of the own and the other's emotions as a way of developing empathy and pro-social behavior. The article finishes by discussing the scope of the process of mediated reading of child literature as a tool that promotes the schooling inclusion and allows the interaction between the cognitive and affective aspects in the formal education.
\end{abstract}

Key words: child literature, mediated reading, basic emotional literacy. 


\section{RESUMO}

A leitura de textos literários na infância permite à criança leitora explorar mundos de ficção nos quais são representadas distintas realidades, caracterizadas por uma série de interações sociais e, portanto, de processos emocionais. No presente artigo, os autores destacam as contribuições da leitura de textos da literatura infantil no desenvolvimento emocional e social das crianças, através de um processo denominado "leitura mediada". Neste processo, o leitor adulto atúa como um mediador da experiência emocional fictícia dos personagens da narrativa, do relato e daqueles processos reais que caracterizam o contexto e a experiência de vida das crianças, sem que a base da narrativa seja direcioná-las para o ensino de determinadas emoções. Desta forma, a leitura mediada de textos literários na infância opera como uma ferramenta de alfabetização emocional, processo que permite o reconhecimento de emoções em si mesmo e nos outros, sendo esta a base da empatia y da conduta pró-social. O artigo finaliza discutindo os alcances do processo de leitura mediada da literatura infantil como uma ferramenta que favorece a inclusão escolar e permite a interação entre os aspectos cognitivos e afetivos na educação formal.

Palavras-chave: literatura infantil, leitura mediada, alfabetização emocional

\section{HACIA UNA RECONSIDERACION DEL MUNDO EMOCIONAL}

Mejorar las habilidades emocionales en el aula se ha convertido en un foco de investigación e intervención reciente (Coppock, 2007; Pianta, 2006). Durante los últimos 10 a 15 años, la literatura asociada a la psicología y educación ha enfatizado la importancia de un adecuado desarrollo social y emocional durante los años preescolares y escolares. Sin embargo, a pesar de la evidencia acumulada sobre su positivo impacto en el desarrollo académico (Denham, 2007), en el desarrollo de habilidades sociales (Richardson, Tolson, Huang \& Lee, 2009), su asociación con el mejoramiento del clima en el aula (Amatruda, 2006; Jennings \& Greenberg, 2009) e incluso los beneficios económicos que implica el invertir en la intervención primaria (Scott, Knapp, Henderson \& Maughan, 2001), las dimensiones afectivas presentes en la dinámica en el aula han sido mantenidas al margen, minimizando su impacto en el desarrollo de los/las estudiantes y su relación con el desempeño académico.

La tradición en el proceso de enseñanza-aprendizaje, muy ligada al quehacer "científico" en psicología (Salovey, Bedell, Detweiler \& Mayer, 2000), hace difícil cambiar la idea de la escuela como un espacio para el aprendizaje definido por un proceso de incorporación de información. Lo cognitivo en esta lógica desplaza la dimensión emocional, que aún dista de ser considerada fundamental en los procesos educativos formales.

Esta separación entre lo afectivo y lo cognitivo tiene profundas raíces que pueden ser ya reconocidas en la filosofía clásica: la Escuela Estoica, por ejemplo, llegó a conceptualizar las emociones "como juicios equivocados sobre el mundo y el lugar de cada uno en él, como formas falsas y destructivas de ver la vida" (Prado, 2006: 16). El método científico "radical" de principios del siglo XX acentúa la división entre lo cognitivo y afectivo. No obstante, a partir del término de la Segunda Guerra Mundial, del "redescubrimiento" de los/las niños/as (Delval, 1998) y de la crisis del pensamiento moderno (Berman, 2004), han surgido intentos por rescatar la emoción como una forma válida de conocer y estar en el mundo (Maturana \& Varela, 1984; Prado, 2006).

En el marco de esta reconsideración del mundo emocional, se han integrado elementos desde diversas disciplinas y recogido las ideas de modelos que integraban la emoción a los distintos procesos de la experiencia humana, especialmente aquellos asociados a la educación formal (Langlois, 2004; Rimé, 2009).

En este "nuevo" contexto educacional se ha comenzado a destacar el rol de la emoción en forma amplia; en poco tiempo se la ha reconsiderado y se hacen intentos por 
integrarla al ámbito educativo (aunque dicha reconsideración aún esté lejos de tener un carácter uniforme y compartido), reconociendo el mundo emocional como una parte de nuestra interacción cotidiana, que desempeña una función central en todos los ámbitos de la vida.

Desde este gran marco, se han levantado distintos modelos de intervención que enfatizan el desarrollo de habilidades emocionales como una forma de prevenir problemas en diversos planos de la interacción de los niños y niñas (Ashiabi, 2000; Merrell, 2000). Hay ejemplos de ello en el plano académico (Bernard, 2006), de las relaciones sociales (Denham, 2007) y familiares (Jennings \& Greenberg, 2009), entre otros*.

Cada modelo de intervención se enmarca en una particular visión del desarrollo emocional; gran parte de ellos ha optado por una visión del apoyo (como intervención) centrada en el individuo (niño o niña) como un agente aislado, señalado como alguien con características idiosincráticas y familiares que lo hacen ser, por su "naturaleza", sujeto de atención especial. Esto se lleva a cabo en procesos que habitualmente culminan con la exclusión del niño/a y de aquellos/as que no han logrado regularse emocionalmente en la interacción escolar (Rimé, 2009). Dicho modelo nos entrega una perspectiva dual, en la que se reconoce el binomio buenos/malos alumnos, como parte de una larga tradición de inclusión/exclusión que aún se mantiene en muchas de nuestras aulas (Marchesi, 2004).

Al mismo tiempo, son muchas las actividades que se presentan como alejadas del contexto regular de los niños y niñas, en settings terapéuticos o de apoyo que en muchas ocasiones distan considerablemente de su cotidianeidad. Así, el niño/a vivencia estos procesos como distantes o ajenos, acentuándose en él/ella la sensación de diferencia y no pertenencia a su grupo.

Los procesos mencionados implican, al menos, dos instancias de quiebre: la primera, asociada a la categoría de "mal alumno" levantada en un primer diagnóstico, y la segunda, asociada a la separación o exclusión como consecuencia de la anterior. Este segundo quiebre es, desde nuestro punto de vista, aún más complejo que el anterior, dado que se excluye al niño/a para ayudarlo/a o, en otras palabras, se le excluye por su propio bien (Marchesi, 2004).

En definitiva, se ha comenzado a reconsiderar el lugar de la afectividad y el desarrollo de habilidades emocionales de los niños, y se ha ofrecido ayuda a quienes tienen dificultades en esta dimensión. Sin embargo, observamos que los mismos mecanismos de apoyo señalados traen consigo los riesgos de constituirse en un contexto artificial, así como la posibilidad de conducir sus acciones hacia la exclusión.

En el presente ensayo reflexionamos sobre una propuesta para el desarrollo de habilidades socioemocionales, fundamentalmente de alfabetización emocional entendida como procesos de interacción, de tal forma que dichas habilidades promuevan en los niños el entendimiento de las emociones propias y ajenas (Nyland, 1999).

Las ideas aquí expuestas se basan en la lectura de literatura infantil como un contexto de interacción regulado o de experiencias mediadas. Así, se presenta una propuesta de trabajo con los niños, en un proceso no forzado, en el que la lectura de literatura Infantil

Una revisión más amplia puede ser encontrada en el libro del Ministerio de Educación, Política Social y Deporte del Gobierno de España (2008). Educación emocional y convivencia en el aula, Secretaría General Técnica, Madrid. 
se produce en forma natural, integrándose al contexto habitual del grupo sin necesidad de generar dinámicas excluyentes.

\section{LA ARQUITECTURA DE FICCION Y EL PROCESO DE LECTURA}

En un trabajo anterior (Munita \& Riquelme, 2009) hemos propuesto la "arquitectura de la ficción" como concepto explicativo del proceso mediante el cual un texto literario se construye, y luego cómo este es reelaborado en la actividad de recepción propia del acto de lectura. Basamos esta idea en la interacción de las nociones "pacto enunciativo" y "mecanismo desacoplador" que, en síntesis, permitirían al niño lector asumir el enunciado literario como una construcción que, pese a su carácter ficcional y a no tener una correspondencia directa con lo "real", tiene la capacidad de representar el mundo cotidiano que le es propio** (Harris, Donnelly, Guz \& Pitt-Watson, 1986).

Como consecuencia de lo anterior, creemos que el aceptar los vehículos imaginarios que entrega la literatura, aun cuando se asuman como enunciados de ficción, permite al lector explorar las emociones y estados mentales de los personajes que viven en el texto. Estos tienen la capacidad de "representar" los estados emocionales propios de la vida humana, actuando en un universo normado por ciertas reglas de verosimilitud que los hacen "creíbles" a los ojos de quien lee. Así, dado el principio de verosimilitud que caracteriza a los buenos relatos, el niño lector puede identificar dichos estados emocionales en un proceso de empatía hacia personajes que viven interacciones y situaciones similares a las suyas. En ellos puede observar sentimientos, valores, conductas que le gustarán o disgustarán (Cervera, 1994), y observará también las causas que llevan a los personajes a actuar o sentir de determinada manera, así como las consecuencias que provocan dichas acciones y/o emociones.

Postulamos entonces el texto literario, y particularmente la narrativa infantil, como una plataforma para la interacción y la alfabetización emocional del niño lector/auditor, pues al considerar el mundo de ficción como una metarrepresentación del mundo real el texto nos permite nombrar y reconocer estados mentales de otros, pero que son atribuibles también a nuestra propia experiencia. Al decir de Michèle Petit: "Los escritores nos ayudan a ponerle un nombre a los estados de ánimo por los que pasamos, a apaciguarlos, a conocerlos mejor, a compartirlos. Gracias a sus historias, nosotros escribimos la nuestra, entre líneas" (1999: 37).

En este marco, toda ficción literaria es una inmersión en el individuo (Machado, 1998), en sus movimientos internos, y el proceso de lectura, por tanto, se constituye como una buena posibilidad de leer mejor el mundo y nuestra particular forma de participación en él.

Muchos autores (Bettelheim, 2004; Cervera, 1994; Sosa, 1982, entre otros) han desarrollado la idea de la literatura como un espacio de "traducción" de los movimientos interiores del niño. De esta manera, por ejemplo, Bruno Bettelheim le otorga una explicación al porqué un niño vuelve una y otra vez al mismo cuento: dicho relato le estaría aportando, a nivel de las estructuras del inconsciente, respuestas a determinadas

Para una mayor profundización de estos conceptos, remitimos al artículo "Arquitectura de la ficción y lector infantil", referenciado en la bibliografía del presente trabajo. 
problemáticas psíquicas e inquietudes psicológicas propias de su etapa de desarrollo. Más allá de este ejemplo y de su particular interpretación, nos interesa reforzar la idea del contacto temprano e intensivo con la literatura como plataforma para atribuir estados emocionales a otros, así como la posibilidad de reconocer y regular los propios.

\section{LA LECTURA MEDIADA DE LITERATURA INFANTIL}

Cuando hablamos de los primeros acercamientos a la literatura, es necesario referirse también a la mediación de la lectura. A la luz del concepto vygotskiano de "mediación", podemos entender al mediador de lectura como un adulto que facilita los primeros acercamientos del niño al libro, en un proceso en el que priman la afectividad y la creación de un momento de lectura acogedor y gratificante. Allí el mediador demuestra su propia dicha de leer para sembrar ese deseo en el otro, en este caso el niño y sus reducidas experiencias de lectura. Es un verdadero "pasador de libros" (Petit, 1999) cuyo objetivo es propiciar un acercamiento afectivo entre el niño y la literatura.

Una de las estrategias de mediación más utilizadas para motivar a los niños hacia la lectura es la del "cuenta cuentos". Entendido de las más diversas formas por los múltiples programas de animación a la lectura surgidos en las últimas décadas, en este artículo lo rescatamos en su acepción más simple: la de un adulto que lee en voz alta un relato para uno o más niños (Munita, 2007). Siguiendo a Cecilia Beuchat, consideramos que "una de las experiencias más interesantes y felices que puede tener un niño es la de escuchar cuentos narrados o leídos por algún adulto" (2006: 15). Así, el mediador, a través de la actividad del cuenta cuentos, provoca una experiencia compartida en la que resulta fundamental el componente afectivo y el placer que produce la lectura, tanto en los niños como en sí mismo. Pues, al entusiasmarse con las historias que narra (Osoro, 2001), el adulto mediador es ya un modelo de lector activo para sus oyentes.

En consecuencia, "contar cuentos es conectar al narrador con el cuento, al narrador con la audiencia, y finalmente a la audiencia con la historia” (Collins \& Cooper, 1997: 41). Dicho proceso de interacción texto-narrador-auditores tiene como objetivo central la conexión del niño con el relato, y esto se logra en buena medida gracias a la capacidad del mediador de "vehiculizar" los sentimientos y atmósferas emocionales propias de la narración, permitiendo así la exploración de estos mundos narrados al niño auditor. A este proceso le llamaremos "lectura mediada".

Para lograr una lectura mediada eficaz, el narrador debe complementar su lectura del texto con otros componentes propios del lenguaje no verbal y paraverbal. En este último, la entonación, el tono y ritmo adecuados serán determinantes para canalizar en forma verosímil y atractiva la trama de la narración. Y en los aspectos no verbales, la kinésica será una excelente manera de regular las acciones del relato y los estados emocionales que este transmite: el significado expresivo de sus gestos y movimientos convertirán al mediador en el puente de acceso a la historia. La tensión o calma de un pasaje determinado, la alegría o tristeza de los personajes o la sorpresa por un giro narrativo inesperado, todo puede manifestarse en la expresión facial y corporal del lector.

En síntesis, en los procesos de lectura mediada convergen diversos lenguajes que, canalizados por la mediación de un adulto, favorecen el acercamiento afectivo del niño al libro y, por extensión, lo introducen en los diversos estados emocionales que habitan los personajes a lo largo de la historia. 


\section{ALGUNAS CONSIDERACIONES SOBRE EL IMPACTO DE LA LECTURA MEDIADA DE LITERATURA INFANTIL}

En la primera parte de este ensayo presentamos un escenario en el que lentamente se han comenzado a incluir los aspectos afectivos en los procesos de formación académica, destacando especialmente las ventajas de esta inclusión para una atención temprana a nivel de prevención inespecífica (académica, social, económica, entre otras). En ese contexto, la alfabetización emocional es un proceso fundamental para esta prevención, aportando al desarrollo de la empatía y de competencias emocionales, así como de las conductas prosociales en los niños (Jennings \& Greenberg, 2009).

En esa línea, varios autores (Colwell \& Hart, 2006; Coppock, 2007; Figueroa, 2008; Richardson, 2009) han planteado numerosas formas de favorecer la alfabetización emocional y el desarrollo social de los niños. Señalan, por ejemplo, la estructuración de ambientes en el aula, la inclusión de familia y comunidad, el trabajo con juegos rítmicos y canciones, grupos temáticos, círculos de tiempo, entre otros. En la mayoría de estos casos el docente opera como monitor de los procesos; en otras palabras, le corresponde actuar como el que enseña el valor correspondiente. También se ha señalado la lectura de cuentos como otra forma de alfabetizarse emocionalmente; en su caso, Figueroa (2008) apunta a aquellos cuentos temáticos, en los cuales se "enseña" explícitamente una emoción que debe ser relatada al niño/a (emoción contenida en la historia, y representada direccionadamente en ella, por ejemplo: un cuento sobre la tristeza).

Al respecto, no sabemos si es posible "enseñar" una emoción determinada en un relato. Esto, en primer lugar, por la naturaleza relacional y contextualizada de los procesos emocionales (Rimé, 2007). Y en segundo término, por el carácter connotativo y polisémico del discurso literario (Sánchez Corral, 1995), cuya relación con la función poética y el pensamiento divergente lo aleja de aquellos textos que "direccionan" un determinado mensaje para el receptor.

Consideramos, entonces, que en la lectura mediada de literatura infantil se exploran conjuntos de narraciones atravesadas por una serie de interacciones cargadas de emoción. En esta exploración el adulto lector opera en la zona de desarrollo proximal del niño lector/ auditor, construyendo junto a él una serie de andamiajes en el lenguaje, que facilitan el reconocimiento de emociones tanto en el mundo de ficción (pero "real”) y en personajes de ficción (pero "reales”) como en los adultos lectores y en sí mismo.

Esta capacidad cognoscitiva de la ficción (Andruetto, 2009), entendida como una "mentira" que permite una nueva mirada sobre el mundo, y en particular sobre los estados emocionales propios de las interacciones de la realidad, se verá favorecida por la lectura de relatos que, lejos de haber sido "manipulados" con ese fin (el cuento "sobre" la tristeza), han sido creados tomando la facultad expresiva de la imagen poética como un reflejo de la serie de interacciones afectivas propias de la vida humana.

A su vez, agregaremos que, en la lectura mediada, el aprendizaje vicario de emociones no se da sólo en el encuentro con el libro en sí mismo, sino que esto se acompaña por la lectura que el mediador hace del texto. En otras palabras, es en el contexto de lectura y diálogo en donde la emoción se sitúa. Como hemos mencionado, el modelo vygotskiano es central en los procesos de lectura mediada; allí el adulto lector entrega la seguridad en la exploración de mundos, que constituyen los conjuntos de narraciones de una serie de interacciones sociales acaecidas en la ficción. 
En este viaje conjunto de exploración de emociones, la alfabetización emocional (como reconocimiento de emociones) es mediada durante el proceso de lectura, y los procesos afectivos son situados en contexto, facilitando al niño la comprensión de los estados emocionales de algún personaje en particular y de la interacción en general.

De esta forma, la narración reúne y entrega una plataforma de exploración. Este proceso básico, de dos o más personas leyendo y comentando un texto literario, integra además los elementos fundamentales para explorar los estados mentales de las otras personas, y facilita a los lectores el compartir dichos estados mentales en un proceso no forzado, integrador y seguro (Rimé, 2009; Riquelme, Henríquez \& Alvarez, 2003).

Es por esto que entendemos la lectura mediada de literatura infantil como una herramienta de exploración de los estados mentales de las demás personas (tales como deseos, emociones y creencias), cuya fortaleza en cuanto prevención inespecífica es no generar dinámicas de exclusión en el aula, sino más bien ser un proceso compartido por todos, y que forma parte del devenir cotidiano del grupo.

Para finalizar, señalaremos que la educación socioafectiva y la académica no deben ser dos prioridades independientes en los procesos formativos de niños y jóvenes. Por el contrario, estas reflexiones nos llevan a pensarlos como un mismo camino que se integra para potenciar un adecuado desarrollo social e individual.

Creemos que dicha integración tiene un buen aliado en la literatura, en el arte de contar con la palabra. Dice la antropóloga francesa Michèle Petit: "Lo que determina la vida del ser humano es en gran medida el peso de las palabras, o el peso de su ausencia. Cuanto más capaz es uno de nombrar lo que vive, más apto será para vivirlo, y para transformarlo. Mientras en el caso contrario, la dificultad de simbolizar puede ir acompañada de una agresividad incontrolable" (1999: 73-74). La buena literatura infantil, en un contexto afectivo de lectura mediada por un adulto, favorecería en el niño la naturalidad de la exploración de contenidos mentales, así como el construir los significados contextualizados de la interacción. Ayudaría, por tanto, a prevenir la agresividad a través de la empatía con personajes que, por los intrincados caminos de la ficción, nos enseñan a nombrar y a nombrarnos.

\section{REFERENCIAS BIBLIOGRAFICAS}

Amatruda, M. (2006). Conflict Resolution and Social Skill Development With Children. Journal of Group Psychotherapy, Psychodrama and Sociometry; 58, 4, p. 168.

Andruetto, M.T. (2009). Hacia una literatura sin adjetivos. Córdoba: Comunicarte.

Ashiabi, G.S. (2000). "Promoting the Emotional Development of Preschoolers". Early Childhood Education Journal. Vol. 28 ( $\left.\mathrm{N}^{\circ} 2\right): 7$.

Beuchat, C. (2006). Narración oral y niños: una alegría para siempre. Santiago de Chile: Universidad Católica de Chile.

Berman, M. (2004). El Reencantamiento del Mundo. $9^{\mathrm{a}}$ ed. Editorial Cuatro Vientos, Chile.

Bernard, M.E. (2006). "It's Time We Teach Social-Emotional Competence As Well As We Teach Academic Competence". Reading \& Writing Quarterly 22: 16.

Bettelheim, B. (2004). Psicoanálisis de los cuentos de hadas. Barcelona: Crítica.

Cervera, J. (1994). La literatura infantil en la construcción de la conciencia del niño. Monteolivete 9-10: 21-36. 
Collins, R. \& Cooper, P. (1997). The power of the story. Scottsdale: Gorsuch Scarisbrick Publishers.

Colwell, M. \& Hart, S. (2006). Emotion framing: does it relate to children's emotion knowledge and social behavior? Early Child Development and Care. Vol. 176, № 6, pp. 591-603.

Coppock, V. (2007). It's good to Talk! A Multidimensional Qualitative Study of the Effectiveness of Emotional Literacy Work in Schools. Children \& Society. Vol. 21, pp. 405-419.

Delval, J. (1998). El desarrollo humano. Madrid: Siglo XXI.

Denham, S.A. (2007). "Dealing With Feelings: How Children Negotiate The Worlds Of Emotions And Social Relationships". Cognitie Creier Comportament / Cognition, Brain, Behavior. Vol. XI ( $\left.\mathrm{N}^{\circ} 1\right)$ : 48.

Doyle, B. \& Bramwell, W. (2006). Promoting emergent literacy and social-emotional learning through dialogic reading. International Reading Association (pp. 554-564).

Figueroa, M. (2008). Building Emotional Literacy: Groundwork to Early Learning. Childhood Education. Vol. 84, № 5, p. 301.

Harris, P., Donnelly, K., Guz, C. \& Pitt-Watson, R. (1986). Children's Understanding of the Distinction between Real and Apparent Emotion. Child Development, Vol. 57, pp. 895-909.

Jennings, J. \& Greenberg, M. (2009). "The Prosocial Classroom: Teacher Social and Emotional Competence in Relation to student and classroom outcomes". Review of Educational Research. Vol. 79 (1): 31.

Langlois, J.H. (2004). "Emotion and Emotion Regulation: From Another Perspective. Child Development, Vol. 75 (2).

Machado, A. (1998). Buenas palabras. Malas palabras. Buenos Aires: Sudamericana.

Marchesi, A. (2004). Qué será de nosotros, los malos alumnos. Madrid: Alianza.

Maturana, H. \& Varela, F. (1984). El árbol del conocimiento. Edit. Universitaria.

Merrell, K.W. (2002). "Social/Emotional Intervention in Schools Current Status, Progress, and Promise". School Pychology Review. Vol. 31 ( $\mathrm{N}^{\circ}$ 2): 6.

Munita, F. (2007). Los caminos del mediador en la animación a la lectura. Novedades Educativas 204-205: 134-139.

Munita, F. y Riquelme, E. (2009). La arquitectura de la ficción y el lector infantil: conjeturas sobre el proceso de articulación en la comprensión literaria. Estudios Pedagógicos. Vol. 35, $\mathrm{N}^{\mathrm{o}} 2$ 261-268.

Nyland, B. (1999). Listening to infants: Emotional literacy and the child care setting. International Journal of Early Childhood; Vol. 31, № 2, p. 99.

Osoro, K. (2001). Leer para fecundar el futuro. Cuatrogatos Revista de Literatura Infantil 7. Extraída de www.cuatrogatos.org. Fecha de acceso: 15 de noviembre de 2009.

Petit, M. (1999). Nuevos acercamientos a los jóvenes y la lectura. México DF: Fondo de Cultura Económica.

Pianta, R.C. (2006). Teacher-child relationships and early literacy. In Dickinson, D.K. \& Neuman, S.B. (eds.), Handbook of Early Literacy Research (Vol. 2) (pp. 149-162). New York: Guilford.

Prado, A. (2006). La interacción entre el relato del mundo televisivo y de la vida real en el proceso de construcción emocional infantil. Facultad de Ciencias de la Comunicación Departamento de Periodismo y Ciencias de la Comunicación. Tesis sin Publicar, presentada para optar al grado de Doctor en Comunicación y Periodismo. Barcelona, Universidad Autónoma de Barcelona.

Richardson, R., Tolson, H., Huang, T.-Y. \& Lee, Y.-H. (2009). "Character Education: Lessons for Teaching Social and Emotional Competence". Children \& Schools. Vol. 31 (2): 8.

Riquelme, E., Henríquez, C. \& Alvarez, C. (2003). Relaciones entre Teoría de la Mente y estilos de Apego. Psykhe. Vol. 12, $\mathrm{N}^{\circ} 1$.

Rimé, B. (2009). "Emotion Elicits the Social Sharing of Emotion: Theory and Empirical Review". Emotion Review 1 (1): 25. 
Salovey, P., Bedell, B., Detweiler, J. \& Mayer, J. (2000). Current Directions in Emotional Intelligence Research. En Gross, J. (2000). Handbook of emotion regulation. New York, NY, The Guilford Press.

Sánchez Corral, L. (1995). Literatura infantil y lenguaje literario. Barcelona: Paidós.

Scott, S., Knapp, M., Henderson, J. \& Maughan, B. (2001). Financial cost of social exclusion: follow up study of antisocial children into adulthood. $B M J ; 323: 1-5$.

Sosa, J. (1982). La literatura infantil. Buenos Aires: Losada. 
